\title{
Finite Corrections to Quark Fragmentation Functions in Perturbative QCD
}

\author{
R. Baier and K. Fey \\ Department of Theoretical Physics, University of Bielefeld, D-4800 Bielefeld, Federal Republic of Germany \\ Received 18 May 1979
}

\begin{abstract}
The finite corrections of order $\alpha_{s}$ in perturbative QCD to the cross-sections for semi-inclusive hadron production from deep inelastic leptonhadron scattering and electron-positron annihilation are calculated. We define the effective quark fragmentation functions via $e^{+} e^{-} \rightarrow$ hadron $+X$ including the finite terms in order to estimate these corrections for the reactions lepton + hadron $\rightarrow$ lepton + hadron + anything. Contrary to the leading term the next-to-leading order term does not factorize into parts depending on the target and the fragment, respectively. For the processes $e+p \rightarrow e+\pi^{ \pm}+X$ and $v+p \rightarrow \mu^{-}+\pi^{ \pm}+X$ the finite corrections of order $\alpha_{s}$ turn out to be at most $20 \%$ in the range of momenta covered by present experiments.
\end{abstract}

\section{Introduction}

We investigate the finite corrections of order $\alpha_{s}$ in perturbative quantumchromodynamics (QCD) to the cross-sections for semi-inclusive hadron production from deep inelastic lepton-hadron scattering and electron-positron annihilation. In the parton model [1] one assumes that the cross-section for the process lepton + hadron $\rightarrow$ lepton + hadron + anything factorizes for each quark coupled to the electromagnetic or weak current into a distribution function $q^{H}(x)$ for finding a quark in the target $H$ and a fragmentation function $D^{H^{\prime}}(z)$ for the scattered quark, which fragments into the final hadron $H^{\prime}$. For electroproduction e.g. one writes

$\frac{1}{d \sigma^{H} / d x} \frac{d \sigma^{H, H^{\prime}}}{d x d z}=\sum_{q} e_{q}^{2} x q_{q}^{H}(x) D_{q}^{H^{\prime}}(z) / \sum_{q} e_{q}^{2} x q_{q}^{H}(x)$.

(The sum is taken over all quark and antiquark flavours).

The variable $x(z)$ is to be interpreted as the fraction of the incoming hadron (outgoing quark) momentum carried by the incoming quark (outgoing hadron) with charge $e_{g}$. The predictive power of the parton model is based on the assumption that the functions $q^{H}(x)$ and $D^{H}(z)$ show scaling behaviour and that they are independent of their environment. This allows to calculate the inclusive hadron production for electron + positron $\rightarrow$ hadron + anything by

$\frac{1}{\sigma_{\text {tot }}} \frac{d \sigma^{H}}{d z}=\sum_{q} e_{q}^{2} D_{q}^{H}(z) / \sum_{\text {quarks. }} e_{q}^{2}$.

In the context of perturbative QCD [2] it was shown that the probabilistic ansatz of the parton model remains preserved [3] in the leading logarithm approximation. The proof is based on the universal factorization of all infrared and mass singularities to all orders in perturbation theory (this factorization is also valid for the non-leading logarithms [3]). The functions $q^{H}$ and $D^{H}$, however, acquire a dependence on the momentum transfer $Q^{2}$. Their $Q^{2}$-evolution is governed by integro-differential equations of the Altarelli and Parisi type [4-6].

The aim of this paper is to calculate the finite (nonleading) terms of order $\alpha_{s}$ for lepton + hadron $\rightarrow$ lepton + hadron + anything and $e^{+} e^{-} \rightarrow$ hadron + anything in order to check their possible importance at moderate values of $Q^{2}$. This kind of corrections has been recently studied for the cross-section of the production of lepton pairs in hadronic collisions $[7,8]$, which is a process like the ones under discussion, for which no light cone techniques are developed. The calculation presented in this paper uses the same assumptions as made in deriving the finite terms for the Drell-Yan process (see [7]), e.g. we neglect interactions with spectator partons and contributions from the two-loop corrections.

We define the effective quark fragmentation functions $D^{H}\left(z, Q^{2}\right)$ by the cross-section $1 / \sigma_{\text {tot }} d \sigma^{H} / d z$ for $e^{+} e^{-} \rightarrow$ hadron + anything including the finite terms such that it keeps the parton model form (1.2). For the effective quark densities $q^{H}\left(x, Q^{2}\right)$ we follow the proposal of Altarelli et al. [7] by demanding that the 
structure function $F_{2}\left(x, Q^{2}\right)$ of deep inelastic lepton hadron scattering is expressed by the $q^{H}\left(x, Q^{2}\right)$ again including the finite terms - as in the parton model. The next-to-leading terms of order $\alpha_{s}$ for the cross-section of lepton + hadron $\rightarrow$ lepton + hadron + anything are then expressed by the quantities $q^{H}\left(x, Q^{2}\right)$ and $D^{H}\left(z, Q^{2}\right)$, but they also depend on the gluon structure - and fragmentation function. The considered correction terms break the factorization property with respect to the $x$ - and $z$-dependence as given by $(1.1)^{1}$.

The paper is organized as follows: In Sect. II the inclusive hadron production in electron-positron annihilation to order $\alpha_{s}$ and our definition of the effective quark fragmentation function are discussed. The hadron production for lepton-hadron scattering is treated in Sect. III. The details of the calculation for the next-to-leading order terms are given for the case of electroproduction. Numerical estimates of the considered corrections are given in Sect. IV with special emphasis on the reactions $e p \rightarrow e \pi^{ \pm} X$, $e p \rightarrow e K^{-} X$ and $\nu p \rightarrow \mu^{-} \pi^{ \pm} X$.

\section{Semi-Inclusive $e+e^{-}$Annihilation and the Effective Quark Fragmentation Function}

The process we are considering is shown in Fig. 1, where a timelike photon with momentum $q$ produces a hadron with momentum $p_{H}$ via an intermediate constituent of momentum $p$; the fragmentation of the constituent is described by a function $D_{\mathrm{c}}^{H}(z)$ (bare fragmentation function), where $z$ is the momentum fraction $z=p_{H} / p$. After integrating with respect to the transverse momentum of the hadron the cross-section is a function of the scaling variable $z_{H}=\frac{2 p_{H} \cdot q}{Q^{2}}$ and of the invariant $Q^{2}=q^{2}$. It is given by the parton model expression [1]

$$
\begin{aligned}
& \frac{d \sigma^{H}}{d z_{H}}\left(z_{H}, Q^{2}\right) \\
& =\sum_{c} \int d z d z_{p} \delta\left(z_{H}-z z_{p}\right) \frac{d \sigma^{c}}{d z_{p}}\left(z_{p}, Q^{2}\right) D_{c}^{H}(z),
\end{aligned}
$$

where $\frac{d \sigma^{c}}{d z_{p}}$ is the differential cross-section for a photon

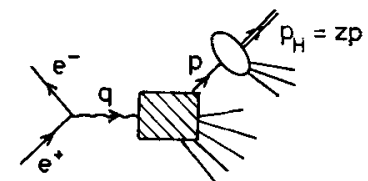

Fig. 1. Parton model description of the process electron + positron $\rightarrow$ hadron $\left(p_{H}\right)+$ anything

\footnotetext{
1 While writing this paper we received a paper by Sakai [9], who discusses the problem of factorization breaking in moments of deep inelastic neutrino hadron production
}
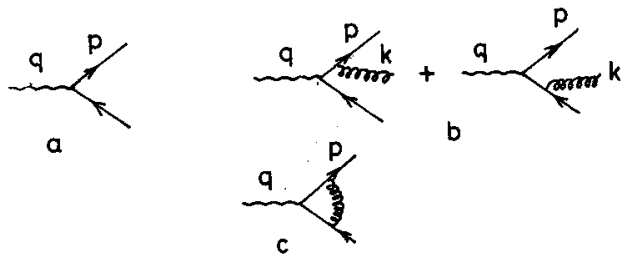

Fig. 2a-c. The diagrams contributing up to order $\alpha_{s}$ to the process shown in Fig. 1. a virtual $\gamma \rightarrow q+\bar{q}$, b virtual $\gamma \rightarrow q+\bar{q}+g$ and c virtual gluon correction to the Born diagram (a). The photon is denoted by a wavy line, quarks by a continuous line and the gluon by a spiral line

decaying into a constituent $c$ (quark $q$, antiquark $\bar{q}$, gluon $g$ ). The variable $z_{p}$ is defined by $z_{p}=\frac{2 p \cdot q}{Q^{2}}$. In lowest order perturbation theory of QCD (Fig. 2a) the cross-section $\frac{d \sigma^{c}}{d z_{p}}$ is given by the parton model formula for the production of a quark-antiquark pair (per coloured quark),

$\frac{d \sigma^{q}}{d z_{q}}=\frac{4 \pi}{3} \frac{\alpha^{2}}{Q^{2}} e_{q}^{2} \delta\left(1-z_{q}\right)$

where $e_{q}$ is the charge of the quark (or antiquark) and $\alpha=1 / 137$. In order to obtain the first order corrections in the strong coupling constant $\alpha_{s}=g^{2} / 4 \pi$ the contributions from the diagrams displayed in Fig. 2 are to be computed, which are due to the emission of a real gluon (Fig. 2b) and due to the interference of the Born term (Fig. 2a) with the virtual gluon diagram (Fig. 2c). Because of the presence of infrared and mass divergencies in the diagrams (Fig. $2 \mathrm{~b}$, c) the gluon momentum is taken off-mass shell by the amount $k^{2}=\mu^{2}, \mu^{2}>0$, whereas the quarks are kept on-shell with zero mass [10]. The explicit calculations are performed in the centre of mass system of the $e^{+} e^{-}$ pair. The Feynman gauge is used. The real diagrams of Fig. $2 \mathrm{~b}$ yield the following differential cross-section [11] (per coloured quark)

$$
\begin{aligned}
& d \sigma^{c}=\frac{4 \pi}{3} e_{q}^{2} \frac{\alpha^{2}}{Q^{2}} \frac{4}{3} \frac{\alpha_{s}}{2 \pi}\left\{\frac{z_{q}^{2}+\left(z_{q}+z_{q}-2\right)^{2}}{\left(1-z_{q}\right)\left(z_{q}+z_{g}-1\right)}\right. \\
& \left.-\frac{\bar{\mu}^{2} z_{q}}{\left(1-z_{q}\right)^{2}}-\frac{\bar{\mu}^{2}}{\left(z_{q}+z_{g}-1\right)^{2}}\right\} d z_{q} d z_{g},
\end{aligned}
$$

where $z_{q}=\frac{2 p \cdot q}{Q^{2}}, z_{g}=\frac{2 k \cdot q}{Q^{2}}$, with $z_{q}+z_{\vec{q}}+z_{g}=2$ and the scaled mass $\bar{\mu}=\mu / \sqrt{Q^{2}}$. The number of colours is set to $N_{c}=3$. Since the cross-section for the inclusive hadron production has the convolution form (2.1), one has to take terms proportional to the gluon mass $\mu$ in (2.3) into account, which give finite contributions in the limit $\mu \rightarrow 0$ after performing moments with respect to $z_{q}$ (or $z_{q}$ ). In order to obtain the expression for $d \sigma^{q} / d z_{q}$ one has to integrate $z_{g}$ over the interval $\left(1-z_{q}\right)+\bar{\mu}^{2} / 1-z_{q} \leqq z_{g} \leqq 1+\bar{\mu}^{2}$ with $z_{q}$ limited by 
$z_{q} \leqq \frac{1}{1+\bar{\mu}^{2}} \simeq 1-\bar{\mu}^{2}$. In the limit of vanishing gluon mass the result is

$$
\begin{aligned}
& \frac{d \sigma^{q}}{d z_{q}}(\text { real })=\frac{4 \pi e_{q}^{2}}{3} \frac{\alpha^{2}}{Q^{2}} \frac{4 \alpha_{s}}{3 \cdot 2 \pi}\left[\ln ^{2} \bar{\mu}^{2} \delta\left(1-z_{q}\right)\right. \\
& +\frac{3}{2} \ln \bar{\mu}^{2} \delta\left(1-z_{q}\right)-\frac{1+z_{q}^{2}}{\left(1-z_{q}\right)_{+}} \ln \bar{\mu}^{2} \\
& +\frac{1+z_{q}^{2}}{\left(1-z_{q}\right)_{+}} \ln \left(1-z_{q}\right)-\frac{3}{2} \frac{1}{1-\left.z_{q}\right|_{+}}+\frac{1+z_{q}^{2}}{1-z_{q}} \\
& \left.\cdot \ln z_{q}+\frac{5}{4} \delta\left(1-z_{q}\right)+\frac{1+z_{q}}{2}\right],
\end{aligned}
$$

with $0 \leqq z_{q} \leqq 1$.

In the intermediate steps of the calculation the following substitutions are used,

$\frac{1}{1-z} \rightarrow \frac{1}{1-\left.z\right|_{+}}-\ln \bar{\mu}^{2} \delta(1-z)$

$\frac{1}{1-z} \ln (1-z) \rightarrow \frac{1}{1-\left.z\right|_{+}} \ln (1-z)-\frac{1}{2} \ln ^{2} \bar{\mu}^{2} \delta(1-z)$

and

$$
\frac{\left(\bar{\mu}^{2}\right)^{n}}{(1-z)^{n+1}} \rightarrow \frac{1}{n} \delta(1-z)
$$

The above relations are derived by considering the moments $\int d z z^{n} f(z)$ in the limit $\mu \rightarrow 0$. All terms vanishing in this limit are neglected. The distribution $1 /(1-z)_{+}$is defined by [4]

$$
\begin{aligned}
& \int_{0}^{1} d z \frac{f(z)}{1-\left.z\right|_{+}}=\int_{0}^{1} d z \frac{f(z)-f(1)}{1-z}, \text { and } \\
& \int_{0}^{1} d z \frac{f(z)}{1-\left.z\right|_{+}} \ln (1-z)=\int_{0}^{1} d z \frac{f(z)-f(1)}{1-z} \ln (1-z) .
\end{aligned}
$$

To the cross-section (2.4) one has to add the interference contribution [10] (Figs. 2a, c),

$$
\begin{aligned}
& \frac{d \sigma^{q}}{d z_{q}}(\text { virtual })=\frac{4 \pi e_{q}^{2}}{3} \frac{\alpha^{2}}{Q^{2}} \frac{4 \alpha_{s}}{3 \cdot 2 \pi} \delta\left(1-z_{q}\right) \\
& \cdot\left[-\ln ^{2} \bar{\mu}^{2}-3 \ln \bar{\mu}^{2}-\frac{7}{2}+\frac{\pi^{2}}{3}\right] .
\end{aligned}
$$

The total contribution for observing a quark (or antiquark) with momentum fraction $z_{q}\left(0 \leqq z_{q} \leqq 1\right)$ is given by

$$
\begin{aligned}
& \frac{d \sigma^{q}}{d z_{q}}=\frac{4 \pi e_{q}^{2}}{3} \frac{\alpha^{2}}{Q^{2}} \\
& \left\{\delta\left(1-z_{q}\right)+\frac{\alpha_{s}}{2 \pi}\left[P_{q q}\left(z_{q}\right) \ln \frac{Q^{2}}{\mu^{2}}+\tilde{d}_{q q}\left(z_{q}\right)\right]\right\},
\end{aligned}
$$

where

$$
P_{q q}(z)=\frac{4}{3}\left[\frac{1+z^{2}}{1-\left.z\right|_{+}}+\frac{3}{2} \delta(1-z)\right] \text {, }
$$

$$
\begin{aligned}
& \tilde{d}_{q q}(z)=\frac{4}{3}\left[\left(\frac{\pi^{2}}{3}-\frac{9}{4}\right) \delta(1-z)+\frac{1+z^{2}}{1-z}\right. \\
& \left.\cdot \ln z-\frac{3}{2} \frac{1}{1-\left.z\right|_{+}}+\frac{1+z^{2}}{1-\left.z\right|_{+}} \ln (1-z)+\frac{1+z}{2}\right] .
\end{aligned}
$$

The leading $\ln ^{2} \bar{\mu}^{2}$ infrared singularities are cancelled between the real and the virtual gluon emission $[3,5]$.

For the differential cross-section of a photon decaying into a gluon with energy fraction $0 \leqq z_{g} \leqq 1$ one has to integrate the cross-section (2.3) with respect to $z_{q}$ in the interval $1-z_{g}+\bar{\mu}^{2} / z_{g} \leqq z_{q} \leqq 1-\bar{\mu}^{2} / z_{g}$. The result is (per coloured quark)

$\frac{1}{2} \frac{d \sigma^{g}}{d z_{g}}=\frac{4 \pi}{3} e_{q}^{2} \frac{\alpha^{2}}{Q^{2}} \frac{\alpha_{s}}{2 \pi}\left[P_{g q}\left(z_{g}\right) \ln \frac{Q^{2}}{\mu^{2}}+d_{g q}\left(z_{g}\right)\right]$,

where

$P_{g q}(z)=\frac{4}{3} \frac{1+(1-z)^{2}}{z}$,

and

$d_{g q}(z)=\frac{4}{3}\left[\frac{1+(1-z)^{2}}{z} \ln z^{2}-2 z\right]$.

We introduce the factor $1 / 2$ into (2.11) because later on we are interested in the correction for each quark or antiquark. In the leading logarithm terms the wellknown transition probability densities $P_{q q}$ and $P_{g q}$ first derived by Altarelli and Parisi [4] appear.

We now define the effective $Q^{2}$-dependent fragmentation function of quark (or antiquark) into hadrons $D_{q}^{H}\left(z, Q^{2}\right)$ to be determined even beyond the leading logarithm approximation by the process $e^{+} e^{-} \rightarrow H+X$ via the standard parton model expression (1.2) (except for the $Q^{2}$-dependence),

$\frac{1}{\sigma_{\text {tot }}} \frac{d \sigma^{e^{+} e^{-} \rightarrow H X}}{d z_{H}}=\sum_{q} e_{q}^{2} D_{q}^{H}\left(z_{H}, Q^{2}\right) / \sum_{\text {quarks }} e_{q}^{2}$.

Applying now this definition we obtain from (2.1) together with the QCD result for the total $e^{+} e^{-} \rightarrow X$ cross-section [12]

$\sigma_{\text {tot }}=3 \sum_{\text {quarks }} \frac{4 \pi}{3} \frac{\alpha^{2}}{Q^{2}} e_{q}^{2}\left(1+\frac{\alpha_{s}\left(Q^{2}\right)}{\pi}\right)$,

the effective quark-fragmentation function $D_{q}^{H}\left(z_{H}, Q^{2}\right)$ in terms of the bare fragmentation functions $D_{q, g}^{z H}(z)$,

$$
\begin{aligned}
& D_{q}^{H}\left(z_{H}, Q^{2}\right) \equiv D_{q}^{H}\left(z_{H}\right)+\frac{\alpha_{s}}{2 \pi} \\
& \cdot \int_{z_{H}}^{1} \frac{d z}{z}\left[\left(P_{q q}\left(\frac{z_{H}}{z}\right) \ln \frac{Q^{2}}{\mu^{2}}+d_{q q}\left(\frac{z_{H}}{z}\right)\right) D_{q}^{H}(z)\right. \\
& \left.+\left(P_{g q}\left(\frac{z_{H}}{z}\right) \ln \frac{Q^{2}}{\mu^{2}}+d_{g q}\left(\frac{z_{H}}{z}\right)\right) D_{g}^{H}(z)\right],
\end{aligned}
$$

where $d_{q q}(z)$ is defined by

$$
d_{q q}(z)=\tilde{d}_{q q}(z)-2 \delta(1-z) \text {. }
$$


In the leading logarithm expansion the $Q^{2}$ dependent fragmentation function $\widetilde{D}_{q}^{H}\left(z_{H}, Q^{2}\right)$ is defined without the finite $d_{q q}$ and $d_{g q}$-terms, respectively. It was conjectured by Georgi and Politzer [5] that $\tilde{D}_{q}^{H}\left(z_{H}, Q^{2}\right)$ satisfies a renormalization group improved evolution equation $[5,6]$

$$
\begin{aligned}
& \frac{d}{d t} \tilde{D}_{q}^{H}\left(z_{H}, t\right)=\frac{\alpha_{s}(t)}{2 \pi} \\
& \cdot \int_{z_{H}}^{1} \frac{d z}{z}\left[P_{q q}\left(\frac{z_{H}}{z}\right) \tilde{D}_{q}^{H}(z, t)+P_{g q}\left(\frac{z_{H}}{z}\right) \tilde{D}_{g}^{H}(z, t)\right],
\end{aligned}
$$

which is the analog of the Altarelli and Parisi evolution equation [4] for the quark distribution function. The variable $t$ is defined by $t=\ln Q^{2} / M^{2}$ where $M$ is the mass corresponding to the renormalization point, $\alpha_{s}(0)=\alpha_{s}$. In Eq. 2.18 corrections of the order $\alpha_{s}^{2}(t)$ in the running coupling constant $\alpha_{s}(t)$ are neglected. The leading logarithm approximation for $\alpha_{s}(t)$ is $\alpha_{s}(t) \simeq \frac{\alpha_{s}}{1+b \alpha_{s} t}$ with $b=\frac{33-2 f}{12 \pi}$, where $f$ is the number of flavour degrees of freedom [2]. It is easy to check that a redefinition of the quark fragmentation function $D_{q}^{H}\left(z_{H}, Q^{2}\right)$ including the finite terms (2.16) again satisfies the evolution equation (2.18) when terms of the order $\alpha_{s}^{2}(t)$ are consistently neglected, since $\frac{d}{d t} \alpha_{s}(t) \propto \alpha_{s}^{2}(t)$. It remains to check that the effective quark decay functions (2.16) obey the momentum sum rule [1]

$\sum_{H}^{1} \int_{0}^{1} d z_{H} z_{H} D_{q}^{H}\left(z_{H}, Q^{2}\right)=1$,

and the isospin sum rule [1]

$$
\sum_{H} \int_{0}^{1} d z_{H} I_{3}^{H} D_{q}^{H}\left(z_{H}, Q^{2}\right)=I_{3}^{q} \text {, }
$$

in order to satisfy the requirement of being consistent definitions. These sum rules (2.20) and (2.21) are assumed to be satisfied by the input-functions $D_{q}^{H}\left(z_{H}\right)$ and $D_{g}^{H}\left(z_{H}\right)$, respectively. In order to prove (2.20) and (2.21) it is convenient to calculate the following moments, the anomalous dimensions [2]

$$
\begin{aligned}
\gamma_{q q}^{n} & =\int_{0}^{1} d z z^{n-1} P_{q q}(z)=\frac{4}{3}\left(-\frac{1}{2}+\frac{1}{n(n+1)}-2 \sum_{j=2}^{n} \frac{1}{j}\right), \\
\gamma_{g q}^{n}=\int_{0}^{1} d z z^{n-1} P_{g q}(z) & =\frac{42+n+n^{2}}{3} n\left(n^{2}-1\right)
\end{aligned}
$$

and the moments of the coefficient functions $d_{q q}$ and $d_{g q}$,

$$
\begin{aligned}
& \delta_{q q}^{n}=\int_{0}^{1} d z z^{n-1} d_{q q}(z) \\
& =\frac{4}{3}\left[\frac{13}{4}-\frac{1}{n^{2}}-\frac{5 n+4}{2 n(n+1)}+\frac{3 n^{2}-n-2}{2 n(n+1)}\right.
\end{aligned}
$$

$\left.\cdot \sum_{j=2}^{n} \frac{1}{j}+2 \sum_{j=2}^{n} \frac{1}{j^{2}}+2 \sum_{j=2}^{n} \frac{1}{j+1} \sum_{k=1}^{j+1} \frac{1}{k}\right]$

and

$\delta_{g q}^{n}=\int_{0}^{1} d z z^{n-1} d_{g q}(z)=-\frac{8 n^{5}+n^{3}+8 n^{2}-2}{3} \frac{n^{2}\left(n^{2}-1\right)^{2}}{}$.

For the validity of (2.21) it is necessary that $\gamma_{q q}^{1}=\delta_{q q}^{1}=0$, whereas for the energy sum rule $(2.20)$ to hold the conditions are $\gamma_{q q}^{2}+\gamma_{g q}^{2}=0$ and $\delta_{q q}^{2}+$ $\delta_{g q}^{2}=0$. All these constraints are satisfied by the moments given in (2.22) and (2.23).

\section{Semi-Inclusive Leptoproduction}

In this section we consider the semi-inclusive leptonhadron scattering shown in Fig. 3 in the deep inelastic limit. The details of the calculation are given for the case of electron (or muon) scattering $e^{-}\left(k_{1}\right)+H\left(p_{H}\right) \rightarrow$ $e^{-}\left(k_{2}\right)+H^{\prime}\left(p_{H}^{\prime}\right)+X$. When the transverse momentum of the outgoing hadron $H^{\prime}$ with momentum $p_{H}^{\prime}$ is not measured the differential cross-section depends on the following variables $[1]$,

$x_{H}=\frac{Q^{2}}{2 p_{H} \cdot q}, \quad y=\frac{p_{H} \cdot q}{p_{H} \cdot k_{1}}$ and $z_{H}=\frac{p_{H}^{\prime} \cdot p_{H}}{p_{H} \cdot q}$,

where $q \equiv k_{1}-k_{2}$ is the momentum of the photon and $Q^{2}=-q^{2}>0$. We neglect masses of the leptons and hadrons. The cross-section is usually written in terms of two structure functions [1],

$$
\begin{aligned}
& \frac{d \sigma^{H, H^{\prime}}}{d x_{H} d y d z_{H}} \\
& =\frac{2 \pi \alpha^{2} s}{Q^{4}}\left[\left(1+(1-y)^{2}\right) F_{2}^{H, H^{\prime}}\left(x_{H}, z_{H}, Q^{2}\right)\right.
\end{aligned}
$$$$
\left.-y^{2} x_{H} F_{L}^{H, H^{\prime}}\left(x_{H}, z_{H}, Q^{2}\right)\right] \text {, }
$$

with $F_{L}^{H, H^{\prime}}=F_{2}^{H, H^{\prime}} / x_{H}-2 F_{1}^{H, H^{\prime}}$, and $s=2 p_{H} \cdot k_{1}=$ $Q^{2} / x_{H} y$. The parton model expression [1] for the process in Fig. 3 is

$$
\begin{aligned}
& d \sigma^{H, H^{\prime}} / d x_{H} d y d z_{H} \\
& =\sum_{a, b} \int d x_{p} d x d z_{p} d z \delta\left(x_{H}-x x_{p}\right) \delta\left(z_{H}-z z_{p}\right) \\
& \cdot q_{a}^{H}(z) \frac{d \sigma^{a, b}}{d x_{p} d y d z_{p}} D_{b}^{H^{\prime}}(z),
\end{aligned}
$$

where $d \sigma^{a, b} / d x_{p} d y d z_{p}$ is the parton cross-section for the initial parton a (quark, antiquark or gluon),

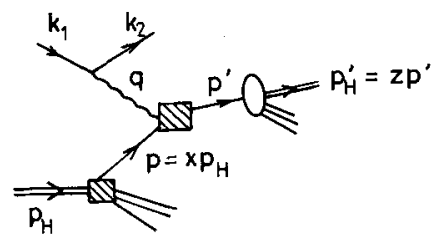

Fig. 3. The parton model description of the process lepton $\left(k_{1}\right)+$ hadron $\left(p_{H}\right) \rightarrow$ lepton $\left(k_{2}\right)+$ hadron $\left(p_{H}^{\prime}\right)+$ anything 
which is scattered to the parton $b$ after interacting with the current. $q_{a}^{H}(x)$ is the bare distribution of parton $a$ in hadron $H$, and $D_{b}^{H^{\prime}}(z)$ is the bare fragmentation function for the transition of parton $b$ into hadron $H^{\prime}$. The parton variables are defined according to (3.1)

$x_{p}=\frac{Q^{2}}{2 p \cdot q}, \quad y=\frac{p \cdot q}{p \cdot k_{1}}$ and $z_{p}=\frac{p \cdot p^{\prime}}{p \cdot q}$,

where $p\left(p^{\prime}\right)$ is the momentum of the incoming (outgoing) parton. The momentum fractions $x$ and $z$ are given by $p=x p_{H}$ and $p_{H}^{\prime}=z p^{\prime}$, respectively. The parton cross-section $d \sigma^{a, b}$ can be decomposed analogously to (3.2) as

$$
\begin{aligned}
& d \sigma^{a, b} / d x_{p} d y d z_{p} \\
& =\frac{2 \pi \alpha^{2} \hat{s}}{Q^{4}}\left[\left(1+(1-y)^{2}\right) F_{2}^{a, b}\left(x_{p}, z_{p}, Q^{2}\right)\right. \\
& \left.-y^{2} x_{p} F_{L}^{a, b}\left(x_{p}, z_{p}, Q^{2}\right)\right],
\end{aligned}
$$

with $\hat{s}=x \cdot s$. In lowest order perturbation theory of QCD (Fig. 4a) only the quarks contribute. The result is

$$
\begin{aligned}
& F_{2}^{q, q}\left(x_{p}, z_{p}, Q^{2}\right)=e_{q}^{2} \delta\left(1-z_{p}\right) \delta\left(1-x_{p}\right), \\
& F_{L}^{q, q}\left(x_{p}, z_{p}, Q^{2}\right)=0 .
\end{aligned}
$$

Using (3.6) the structure functions defining the total inclusive cross-section for $e^{-}+$hadron $\rightarrow e^{-}+X$ have the form

$F_{2}^{H}\left(x_{H}, Q^{2}\right)=\sum_{q} e_{q}^{2} x_{H} q_{q}^{H}\left(x_{H}\right), \quad F_{L}^{H}\left(x_{H}\right)=0$.

(In (3.7) and in the following $\sum_{q}$ means the sum over quark and antiquark flavours). These are the usual parton model results [1].

In first order in the strong coupling constant $\alpha_{s}$ the contributions shown in Figs. $4 b-d$ are to be calculated; virtual $\gamma+q \rightarrow q+g$, where either the quark or the gluon can fragment into the hadron $H^{\prime}$, and virtual $\gamma+g \rightarrow q+\bar{q}$ with the quark (or the antiquark) fragmenting into $H^{\prime}$. For the elastic case virtual $\gamma+q \rightarrow q$ the interference of the Born term (Fig. 4a) with the graph in Fig. $4 \mathrm{c}$ has to be taken into account. The same technical assumptions are made in the evaluation of the diagrams (Fig. 4) in order to be consistent with the calculation of Sect. II. The
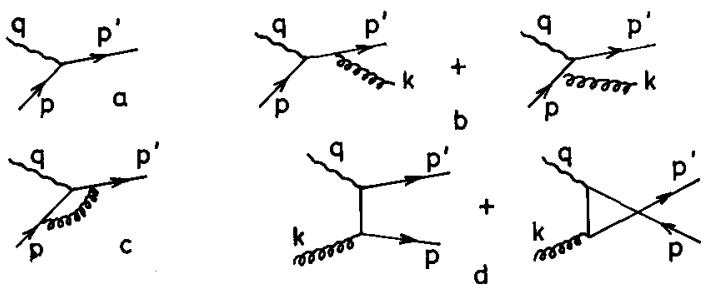

Fig. 4a-d. The diagrams contributing up to order $\alpha_{s}$ to electroproduction; a virtual $\gamma+q(p) \rightarrow q\left(p^{\prime}\right)$, b virtual $\gamma+q(p) \rightarrow$ $q\left(p^{\prime}\right)+g(k)$, c virtual gluon correction to the Born diagram (a), d virtual $\gamma+g(k) \rightarrow q\left(p^{\prime}\right)+\bar{q}(p)$. The photon is denoted by a wavy line, quarks by a continuous line and the gluon by a spiral line explicit calculations are performed in the Breit frame [1].

When triggering on the outgoing quark with momentum $p^{\prime}\left(z_{q}=\frac{p \cdot p^{\prime}}{p \cdot q}\right)$ we obtain from the diagrams with real gluon emission

$$
\begin{aligned}
& F_{2}^{q, q}\left(x_{q}, z_{q}, Q^{2}\right) / x_{q}=e_{q}^{2} \frac{\alpha_{s}}{2 \pi} \frac{4}{3}\left[\frac{\left(1-z_{q}-x_{q}\right)^{2}+1}{\left(1-x_{q}\right)\left(1-z_{q}-x_{q} \bar{\mu}^{2}\right)}\right. \\
& +6 x_{q} z_{q}-\bar{\mu}^{2} x_{q}\left(\frac{1}{\left(1-x_{q}\right)^{2}}-\frac{1-x_{q}\left(1+\bar{\mu}^{2}\right)-z_{q}}{\left(1-z_{q}-x_{q} \bar{\mu}^{2}\right)^{2}}\right. \\
& \left.\left.+\frac{\left(1+z_{q}\right)+\left(1-x_{q}\right)}{\left(1-x_{q}\right)\left(1-z_{q}-x_{q} \bar{\mu}^{2}\right)}\right)\right] \\
& F_{L}^{q, q}\left(x_{q}, z_{q}, Q^{2}\right)=e_{q}^{2} \frac{\alpha_{s}}{2 \pi} \frac{4}{3} 4 z_{q} x_{q}
\end{aligned}
$$

where $\bar{\mu}^{2}=\mu^{2} / Q^{2}>0$. The boundaries of the variables $x_{q}$ and $z_{q}$ are given by $0 \leqq x_{q} \leqq 1 / 1+\bar{\mu}^{2}$ and $0 \leqq z_{q} \leqq$ $1-x_{q} \bar{\mu}^{2} / 1-x_{q}$. In the limit $\mu^{2} \rightarrow 0$ the above expressions agree with [13]. In order to calculate the expressions for $F_{2, L}^{q, q}$ in the limit $\bar{\mu}^{2} \rightarrow 0$ one has to handle the infrared and mass divergencies, which are generated in the limit $z_{q} \rightarrow 1$ and $x_{q} \rightarrow 1$ (soft gluon emission) and $z_{q} \rightarrow 1$ (hard gluon emission parallel to the incoming quark) or $x_{q} \rightarrow 1$ (hard gluon emission parallel to the outgoing quark). Because of the convolutions (3.3) the explicit procedure is to define

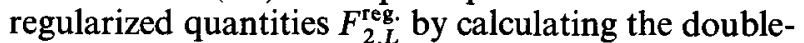
moments with respect to $x_{q}$ and $z_{q}$ and by demanding

$\lim _{\tilde{\mu}^{2} \rightarrow 0} \int_{0}^{1 / 1+\bar{\mu}^{2}} d x_{q} \int_{0}^{1-x_{q} \tilde{\mu}^{2} / 1-x_{q}} d z_{q} x_{q}^{m} z_{q}^{n} F_{2, L}^{q, q}$
$=\int_{0}^{1} d x_{q} \int_{0}^{1} d z_{q} x_{q}^{m} z_{q}^{n} F_{2, L}^{r e g}$

+ singular terms proportional to $\ln \bar{\mu}^{2}$ and $\ln ^{2} \bar{\mu}^{2}$.

Again terms, which vanish in the limit $\bar{\mu}^{2} \rightarrow 0$ are neglected. In addition to the substitutions (2.5) one obtains the following prescriptions valid for the double integrals in (3.10),

$$
\begin{aligned}
& \frac{1}{1-z-x \bar{\mu}^{2}} \rightarrow \frac{1}{1-\left.z\right|_{+}}-\ln \frac{x^{2}}{1-x} \delta(1-z) \\
& -\ln \bar{\mu}^{2} \delta(1-z), \\
& \frac{1}{(1-x)\left(1-z-x \bar{\mu}^{2}\right)} \rightarrow \frac{1}{(1-x)_{+}(1-z)_{+}} \\
& +\delta(1-x) \frac{\ln (1-z)}{1-\left.z\right|_{+}}-\left(\frac{\ln x^{2}}{1-x}-\frac{\ln (1-x)}{(1-x)_{+}}\right) \delta(1-z) \\
& -\ln \bar{\mu}^{2}\left(\frac{\delta(1-x)}{(1-z)_{+}}+\frac{\delta(1-z)}{(1-x)_{+}}\right) \\
& +\frac{1}{2} \ln ^{2} \bar{\mu}^{2} \delta(1-x) \delta(1-z), \\
& \frac{\bar{\mu}^{2}}{(1-x)^{2}} \rightarrow \delta(1-x)(1-z) \text { and } \frac{\bar{\mu}^{2} x^{2}}{\left(1-z-x \bar{\mu}^{2}\right)^{2}} \\
& \rightarrow \delta(1-z)(1-x) .
\end{aligned}
$$


Adding the contribution from the interference of the graph in Fig. $4 c$ with the Born term,

$$
\begin{aligned}
& \left.F_{2}^{q, q}\left(x_{q}, z_{q}, Q^{2}\right)\right|_{\text {virtual }}=-e_{q}^{2} \frac{4 \alpha_{s}}{3 \cdot 2 \pi} \delta\left(1-x_{q}\right) \delta\left(1-z_{q}\right) \\
& \cdot\left[\ln ^{2} \bar{\mu}^{2}+3 \ln \bar{\mu}^{2}+\frac{7}{2}+\frac{2 \pi^{2}}{3}\right],
\end{aligned}
$$

one obtains finally the structure functions for the transition of quark into quark with $0 \leqq x_{q} \leqq 1$ and $0 \leqq z_{q} \leqq 1$,

$$
\begin{aligned}
& F_{2}^{q, q}\left(x_{q}, z_{q}, Q^{2}\right) / x_{q}=e_{q}^{2} \frac{\alpha_{s}}{2 \pi}\left[\operatorname { l n } \frac { Q ^ { 2 } } { \mu ^ { 2 } } \left(P_{q q}\left(x_{q}\right) \delta\left(1-z_{q}\right)\right.\right. \\
& \left.\left.+P_{q q}\left(z_{q}\right) \delta\left(1-x_{q}\right)\right)+f_{q q}\left(x_{q}, z_{q}\right)\right],
\end{aligned}
$$

where

$$
\begin{aligned}
& f_{q q}\left(x_{q}, z_{q}\right)=\frac{4}{3}\left[-\left(\frac{7}{2}+\frac{2 \pi^{2}}{3}\right) \delta\left(1-x_{q}\right) \delta\left(1-z_{q}\right)\right. \\
& +\delta\left(1-x_{q}\right)\left(z_{q}-1+\frac{1+z_{q}^{2}}{1-\left.z_{q}\right|_{+}} \ln \left(1-z_{q}\right)\right) \\
& +\delta\left(1-z_{q}\right)\left(\frac{1+x_{q}^{2}}{1-\left.x_{q}\right|_{+}} \ln \left(1-x_{q}\right)-\frac{1+x_{q}^{2}}{1-x_{q}}\right. \\
& \left.\cdot \ln x_{q}^{2}+x_{q}-1\right)+6 x_{q} z_{q}+1+\frac{1+z_{q}}{1-\left.x_{q}\right|_{+}} \\
& \left.-\frac{z_{q}+x_{q}}{1-z_{q} \mid}+\frac{2 z_{q}^{2}}{\left(1-x_{q}\right)_{+}\left(1-z_{q}\right)_{+}}\right] .
\end{aligned}
$$

The longitudinal part $F_{L}^{q, q}$ (3.9) is independent of mass divergencies. The density $P_{a q}$ is the same as defined in (2.9). One observes that the soft gluon singularity is cancelled. The finite term $f_{q q}\left(x_{q}, z_{q}\right)$ does not factorize with respect to its $x_{q}$ and $z_{q}$ dependence in the way the divergent terms do.

The case where the hard gluon $\left(z_{g}>0\right)$ is supposed to fragment into the hadron $H^{\prime}$ can be obtained immediately from (3.12) and (3.13) by substituting $z_{q} \rightarrow 1-z_{g}$ and by dropping the terms proportional to $\delta\left(z_{g}\right)$

$$
\begin{aligned}
& \frac{F_{2}^{q, g}}{x_{q}}\left(x_{q}, z_{g}, Q^{2}\right)=e_{q}^{2} \frac{\alpha_{s}}{2 \pi} \\
& \cdot\left[\ln \frac{Q^{2}}{\mu^{2}} P_{q g}\left(z_{g}\right) \delta\left(1-x_{q}\right)+f_{q g}\left(x_{q}, z_{g}\right)\right],
\end{aligned}
$$

where $P_{q g}(z)=P_{q q}(1-z)$ and $f_{q g}(z)=f_{q q}(1-z)$.

We now consider the contributions to the semiinclusive cross-section in the case of an incoming gluon (Fig. 4d). Here the mass divergencies for the hard gluon to be parallel to either of one of the outgoing quarks are regularized by putting $k^{2}=-\mu^{2}$, $\mu^{2}>0$. We find

$$
\begin{aligned}
& F_{2}^{g, q}\left(x_{g}, z_{q}, Q^{2}\right) / x_{g}=e_{q}^{2} \frac{\alpha_{s}}{2 \pi} \frac{1}{2}\left[\frac{1-z_{q}-2 x_{g} \bar{\mu}^{2}}{z_{q}+x_{g} \bar{\mu}^{2}}\right. \\
& +\frac{z_{q}}{1-z_{q}-x_{g} \bar{\mu}^{2}}-2\left(1-x_{g}-x_{g} \bar{\mu}^{2}\right)\left(\frac{1}{z_{q}+x_{g} \mu^{2}}\right.
\end{aligned}
$$

$$
\begin{aligned}
& \left.+\frac{1}{1-z_{q}-x_{g} \bar{\mu}^{2}}-\frac{1-x_{g}-x_{g} \bar{\mu}^{2}}{\left(z_{q}+x_{g} \bar{\mu}^{2}\right)\left(1-z_{q}-x_{g} \bar{\mu}^{2}\right)}\right) \\
& +12 x_{g}\left(1-x_{g}\right)+\bar{\mu}^{2} x_{g}\left(1-x_{g}-x_{g} \bar{\mu}^{2}\right) \\
& \cdot\left(\frac{1}{\left(z_{q}+x_{g} \bar{\mu}^{2}\right)^{2}}+\frac{1}{\left(1-z_{q}-x_{g} \bar{\mu}^{2}\right)^{2}}\right) \\
& \left.-\bar{\mu}^{2} x_{g} \frac{1-z_{q}-2 x_{g} \bar{\mu}^{2}}{\left(z_{q}+x_{g} \bar{\mu}^{2}\right)^{2}}-\bar{\mu}^{2} x_{g} \frac{z_{q}}{\left(1-z_{q}-x_{g} \bar{\mu}^{2}\right)^{2}}\right],
\end{aligned}
$$

$\left(\right.$ where $\left.x_{g}=Q^{2} / 2 k \cdot q, z_{q}=\frac{k \cdot p^{\prime}}{k \cdot q}\right)$, and

$F_{L}^{g q}\left(x_{g}, z_{q}, Q^{2}\right)=e_{q}^{2} \frac{\alpha_{s}}{2 \pi} \frac{1}{2} 8 x_{g}\left(1-x_{g}\right)$.

For $\bar{\mu}^{2} \rightarrow 0$ the boundaries are given by $0 \leqq x_{q} \leqq 1-\bar{\mu}^{2}$ and $-\bar{\mu}^{2} x_{g}\left(1-x_{g}\right) \leqq z_{q} \leqq 1-\bar{\mu}^{2} x_{g}\left(1+x_{g}\right)$.

Again for $\bar{\mu}^{2} \rightarrow 0$ there is agreement with [13]. The regularization procedure is performed analogously to (3.10), but here one calculates the double moments with respect to $x_{g}$ and $z_{q}$. After using the following substitutions

$\frac{1}{1-z_{q}-x_{g} \bar{\mu}^{2}} \rightarrow \frac{1}{1-\left.z_{q}\right|_{+}}-\delta\left(1-z_{q}\right) \ln x_{g}^{2}$

$-\ln \bar{\mu}^{2} \delta\left(1-z_{q}\right)$,

$\frac{1}{\left(z_{q}+x_{g} \vec{\mu}^{2}\right)\left(1-z_{q}-x_{g} \bar{\mu}^{2}\right)} \rightarrow \frac{1}{z_{q} l_{+}\left(1-z_{q}\right)_{+}}$

$-\left(\delta\left(z_{q}\right)+\delta\left(1-z_{q}\right)\right)\left(\ln x_{q}^{2}+\ln \bar{\mu}^{2}\right)$,

$\frac{\bar{\mu}^{2}}{\left(1-z_{q}-x_{g} \bar{\mu}^{2}\right)^{2}} \rightarrow \frac{1}{x_{g}^{2}} \delta\left(1-z_{q}\right)$

we obtain

$$
\begin{aligned}
& \frac{F_{2}^{g, q}\left(x_{g}, z_{q}, Q^{2}\right)}{x_{g}} \\
& =e_{q}^{2} \frac{\alpha_{s}}{2 \pi}\left[\ln \frac{Q^{2}}{\mu^{2}} P_{q g}\left(x_{g}\right)\left(\delta\left(z_{q}\right)+\delta\left(1-z_{q}\right)\right)+f_{g q}\left(x_{g}, z_{q}\right)\right],
\end{aligned}
$$

and $0 \leqq x_{g}, z_{q} \leqq 1$, where

$$
\begin{aligned}
& P_{q g}(x)=\frac{1}{2}\left(x^{2}+(1-x)^{2}\right), \\
& f_{g q}(x, z)=\frac{1}{2}\left[-\left(x^{2}+(1-x)^{2}\right) \ln x^{2}(\delta(z)+\delta(1-z))\right. \\
& \left.-\delta(z)-\delta(1-z)+\frac{x^{2}+(1-x)^{2}}{(1-z)_{+}(z)_{+}}-2+12 x(1-x)\right] .
\end{aligned}
$$

Before finally discussing the hadron cross-section (3.2) the total inclusive leptoproduction including the $\alpha_{s}$-contributions is presented. The parton model expression for $F_{2}^{H}(3.7)$ becomes modified by

$$
\begin{aligned}
& F_{2}^{H}\left(x_{H}, Q^{2}\right)=\sum_{q} e_{q}^{2} x_{H}\left\{q_{q}^{H}\left(x_{H}\right)\right. \\
& +\frac{\alpha_{s}}{2 \pi} \int_{x_{H}}^{1} \frac{d x}{x}\left[\left(P_{q q}\left(\frac{x_{H}}{x}\right) \ln \frac{Q^{2}}{\mu^{2}}+f_{q}\left(\frac{x_{H}}{x}\right)\right) q_{q}^{H}(x)\right.
\end{aligned}
$$




$$
\left.\left.+\left(P_{q g}\left(\frac{x_{H}}{x}\right) \ln \frac{Q^{2}}{\mu^{2}}+f_{g}\left(\frac{x_{H}}{x}\right)\right) g^{H}(x)\right]\right\},
$$

where $q_{q}^{H}(x)$ and $g^{H}(x)$ are the bare quark (or antiquark) and gluon distribution functions for the hadron $H$. The finite terms $f_{q, g}$ are derived as follows

$$
\begin{aligned}
& f_{q}(x)=\int_{0}^{1} d z_{q} f_{q q}\left(x, z_{q}\right)=\frac{4}{3}\left[\delta(1-x)\left(-\frac{2 \pi^{2}}{3}-\frac{9}{4}\right)\right. \\
& +1+4 x-\frac{3}{2} \frac{1}{1-\left.x\right|_{+}}+\frac{1+x^{2}}{1-\left.x\right|_{+}} \ln (1-x) \\
& \left.-\frac{1+x^{2}}{1-x} \ln x^{2}\right]
\end{aligned}
$$

and

$$
\begin{aligned}
& f_{g}(x)=\frac{1}{2} \int_{0}^{1} f_{g q}\left(x, z_{q}\right) d z_{q} \\
& =\frac{1}{2}\left[-\left(x^{2}+(1-x)^{2}\right) \ln x^{2}-2+6 x(1-x)\right] .
\end{aligned}
$$

The factor $1 / 2$ is taken because we are summing over quarks and antiquarks in (3.19) and the diagrams (Fig. 4d) are only to be counted once. Our results for $f_{q, g}(x)$ can be compared with the ones of [7]. There is agreement for $f_{q}$, but not for $f_{q}$, but in the calculation of $f_{q}$ in [7] the quarks are set off-shell.

For the following we adopt the same convention as in Ref. 7 in order to define the $Q^{2}$-dependent effective quark distributions $q\left(x_{H}, Q^{2}\right)$ by assuming that the expression for $F_{2}^{H}\left(x_{H}, Q^{2}\right)$ has the same form as in the parton model (3.7) even when the finite terms of $\alpha_{s}$ are included

$F_{2}^{H}\left(x_{H}, Q^{2}\right)=\sum_{q} e_{q}^{2} x_{H} q_{q}^{H}\left(x_{H}, Q^{2}\right)$.

This gives the relation

$$
\begin{aligned}
& q_{q}^{H}\left(x_{H}, Q^{2}\right) \equiv q_{q}^{H}\left(x_{H}\right) \\
& +\frac{\alpha_{s}}{2 \pi} \int_{x_{H}}^{1} \frac{d x}{x}\left[\left(P_{q q}\left(\frac{x_{H}}{x}\right) q_{q}^{H}(x)+P_{q g}\left(\frac{x_{H}}{x}\right) g^{H}(x)\right)\right. \\
& \left.\cdot \ln \frac{Q^{2}}{\mu^{2}}+\left(f_{q}\left(\frac{x_{H}}{x}\right) q_{q}^{H}(x)+f_{g}\left(\frac{x_{H}}{x}\right) g^{H}(x)\right)\right] .
\end{aligned}
$$

One has to note, as discussed by Altarelli et al. [7], that although the finite terms proportional to $f_{q, q}$ are incorporated in the definition of $q_{q}^{H}\left(x_{H}, Q^{2}\right)$, the $Q^{2}$-dependence of $q_{q}^{H}\left(x_{H}, Q^{2}\right)$ is at order $\alpha_{s}\left(Q^{2}\right)$ determined by the Altarelli-Parisi equation [4]

$$
\begin{aligned}
& \frac{d}{d t} q^{H}\left(x_{H}, t\right)=\frac{\alpha_{s}(t)}{2 \pi} \int_{x_{H}}^{1} \frac{d x}{x}\left[q^{H}(x, t) P_{q q}\left(\frac{x_{H}}{x}\right)\right. \\
& \left.+g^{H}(x, t) P_{q q}\left(\frac{x_{H}}{x}\right)\right] .
\end{aligned}
$$

The definition of $q^{H}\left(x, Q^{2}\right)$ given by (3.21) is consistent with the parton model interpretation of $F_{2}^{H}\left(x, Q^{2}\right)$ because it satisfies the charge sum rule [1]

$$
\begin{aligned}
& \int_{0}^{1} d x_{H}\left[q^{H}\left(x_{H}, Q^{2}\right)-\bar{q}^{H}\left(x_{H}, Q^{2}\right)\right] \\
& =\int_{0}^{1} d x_{H}\left[q^{H}\left(x_{H}\right)-\bar{q}^{H}\left(x_{H}\right)\right]
\end{aligned}
$$

$=$ number of valence quarks in hadron $H$,

since $\gamma_{q q}^{1}=0$ and $\int_{0}^{1} d z f_{q}(z)=0$.

After having discussed the prescriptions of measuring effective quark-fragmentation functions $D_{q}^{H}\left(z_{H}, Q^{2}\right)$ and quark structure functions $q^{H}\left(x_{H}, Q^{2}\right)$ we can finally calculate the semi-inclusive crosssection $\frac{d \sigma^{H, H^{\prime}}}{d x_{H} d y d z_{H}}$ in terms of these functions. With the help of (2.16) and (3.21) we eliminate the bare functions $D_{q}^{H}\left(z_{H}\right)$ and $q^{H}\left(x_{H}\right)$ and we perform the sum over the contributions given by (3.9), (3.13), (3.14), (3.16) and (3.18) keeping all terms of order $\alpha_{s}$ consistently. Because of the universality of the $\ln Q^{2} / \mu^{2}$ terms the final result for the semi-inclusive cross-section $d \sigma^{H, H^{\prime}} / d x_{H} d y d z_{H}$ is now free of mass singularities [3], since they are all absorbed into the effective parton distribution and fragmentation functions, respectively. As a final result of our analysis the corrections of order $\alpha_{s}$ (beyond the leading logarithm) can be summarized as,

$$
\begin{aligned}
& F_{2}^{H, H^{\prime}}\left(x_{H}, z_{H}, Q^{2}\right)=\sum_{q} e_{q}^{2} x_{H} q_{q}^{H}\left(x_{H}, Q^{2}\right) D_{q}^{H^{\prime}}\left(z_{H}, Q^{2}\right) \\
& +F_{\text {corr. }}^{H, H^{\prime}}\left(x_{H}, z_{H}, Q^{2}\right), \\
& F_{\text {corr. }}^{H, H^{\prime}}=\frac{\alpha_{s}\left(Q^{2}\right)}{2 \pi} \frac{4}{3} \sum_{q} e_{q}^{2} x_{H} \\
& \cdot \int_{x_{H}}^{1} \frac{d x}{x} \int_{z_{H}}^{1} \frac{d z}{z}\left[q_{q}^{H}\left(x, Q^{2}\right) D_{q}^{H^{\prime}}\left(z, Q^{2}\right) c_{q q}\left(\frac{x_{H}}{x}, \frac{z_{H}}{z}\right)\right. \\
& +q_{q}^{H}\left(x, Q^{2}\right) D_{g}^{H^{\prime}}\left(z, Q^{2}\right) c_{q g}\left(\frac{x_{H}}{x}, \frac{z_{H}}{z}\right) \\
& \left.+g^{H}\left(x, Q^{2}\right) D_{q}^{H^{\prime}}\left(z, Q^{2}\right) c_{g q}\left(\frac{x_{H}}{x}, \frac{z_{H}}{z}\right)\right],
\end{aligned}
$$

where

$$
\begin{aligned}
& c_{q q}(x, z)=\left(-\frac{\pi^{2}}{3}+\frac{5}{2}\right) \delta(1-x) \delta(1-z) \\
& +\delta(1-x)\left[-\frac{1+z^{2}}{1-z} \ln z+\frac{3}{2} \frac{1}{(1-z)_{+}}-\frac{3-z}{2}\right] \\
& +\delta(1-z)\left[-z-3 x+\frac{3}{2} \frac{1}{1-\left.x\right|_{+}}\right] \\
& +1+\frac{2 z^{2}}{(1-z)_{+}(1-x)_{+}}+\frac{1+z}{(1-x)_{+}}-\frac{z+x}{(1-z)_{+}}+6 x z, \\
& c_{q g}(x, z)=\delta(1-x)\left[-\frac{1+(1-z)^{2}}{z} \ln z+z\right] \\
& +2+6 x(1-z)-\frac{1+x}{z}+\frac{2(1-z)^{2}}{z(1-x)_{+}}+\frac{2-z}{(1-x)_{+}},
\end{aligned}
$$


and

$C_{g q}(x, z)=\frac{3}{8} \delta(1-z)\left(6 x^{2}-6 x+1\right)$

$+\frac{3}{8} \frac{x^{2}+(1-x)^{2}}{(1-z)_{+} z}-\frac{3}{4}+\frac{9}{2} x(1-x)$.

In the derivation of (3.24) the bare gluon distribution $g^{H}\left(x_{H}\right)$ and fragmentation function $D_{g}^{H}\left(z_{H}\right)$ can be replaced by their $Q^{2}$-dependent effective functions $g^{H}\left(x_{H}, Q^{2}\right)$ and $D_{g}^{H}\left(z_{H}, Q^{2}\right)$, respectively, since they enter in the terms, which are already of the order $\alpha_{s}$. The coupling constant $\alpha_{s}$ is to be evaluated at $\alpha_{s}\left(Q^{2}\right)$ in order to make the perturbation series for the finite terms converge rapidly [3]. In order to evaluate the above integrals, one uses

$\int_{x_{H}}^{1} \frac{d x}{x} \frac{f\left(\frac{x_{H}}{x}\right)}{\left(1-\frac{x_{H}}{x}\right)_{+}}$
$=\int_{x_{H}}^{1} \frac{d x}{x} \frac{f\left(\frac{x_{H}}{x}\right)-f(1)}{\left(1-\frac{x_{H}}{x}\right)}+f(1) \ln \frac{1-x_{H}}{x_{H}}$.

The longitudinal part of the cross-section is found to be

$F_{L}^{H, H^{\prime}}\left(x_{H}, z_{H}, Q^{2}\right)=\frac{\alpha_{s}\left(Q^{2}\right)}{2 \pi} \frac{16}{3} \sum_{q} e_{q}^{2}$

$\cdot \int_{x_{\mathrm{H}}}^{1} \frac{d x}{x} \int_{z_{H}}^{1} \frac{d z}{z}\left\{q_{q}^{H}\left(x, Q^{2}\right) D_{q}^{H^{\prime}}\left(z, Q^{2}\right) \frac{x_{H}}{x} \frac{z_{H}}{z}\right.$

$+q_{q}^{H}\left(x, Q^{2}\right) D_{g}^{H^{\prime}}\left(z, Q^{2}\right) \frac{x_{H}}{x}\left(1-\frac{z_{H}}{z}\right)$

$\left.+\frac{3}{4} g^{H}\left(x, Q^{2}\right) D_{q}^{H^{\prime}}\left(z, Q^{2}\right) \frac{x_{H}}{x}\left(1-\frac{x_{H}}{x}\right)\right\}$.

From (3.24), (3.26) one can rederive (as a consistency check) the totally inclusive cross-section by evaluating the sum rule

$\sum_{H^{\prime}}^{1} \int_{0}^{1} d z_{H} z_{H} \frac{d \sigma^{H, H^{\prime}}}{d x_{H} d y d z_{H}}=\frac{d \sigma^{H}}{d x_{H} d y}$,

with

$\sum_{H^{\prime}}^{1} \int_{0}^{1} d z_{H^{\prime}} z_{H} D_{q, g}^{H^{\prime}}\left(z_{H}, Q^{2}\right)=1$

Indeed the contribution to $F_{2}^{H, H^{\prime}}$ of order $\alpha_{s}\left(Q^{2}\right)$ vanishes, $\sum_{H^{\prime}}^{1} \int_{0}^{1} d z_{H} z_{H} F_{\text {corr. }}^{H, H^{\prime}}=0$. For the longitudinal part we obtain

$F_{L}^{H}\left(x_{H}, Q^{2}\right)=\sum_{H^{\prime}} \int_{0}^{1} d z_{H} z_{H} F_{L}^{H, H^{\prime}}\left(x_{H}, z_{H}, Q^{2}\right)$

$$
\begin{aligned}
& =\frac{\alpha_{s}\left(Q^{2}\right)}{2 \pi} x_{H} \int_{x_{H}}^{1} \frac{d x}{x^{3}}\left[\frac{8}{3} F_{2}^{H}\left(x, Q^{2}\right)\right. \\
& \left.+2 x \sum_{q} e_{q}^{2}\left(1-\frac{x_{H}}{x}\right) g^{H}\left(x, Q^{2}\right)\right],
\end{aligned}
$$

which agrees with the result of [7].

\section{Numerical Estimates}

A numerical evaluation of the finite corrections of order $\alpha_{s}$ to the semi-inclusive hadron production derived in the previous chapter (3.24) and (3.26) requires a specification of the quark and gluon structure and fragmentation functions. For the estimates given below the parametrizations of the functions $q\left(x, Q^{2}\right), g\left(x, Q^{2}\right)$ and $D_{q, g}^{\pi, K}$, including their $Q^{2}$-dependence, are used as they are discussed in Ref. 14. In Fig. $(5 \mathrm{a}, \mathrm{b})$ we plot the $Q^{2}$-dependence of the relative correction to the structure function $F_{2}^{H, H^{\prime}}$ for a proton target $H=p$,

$F_{\text {corr. }}^{p, H^{\prime}}\left(x_{H}, z_{H}, Q^{2}\right) / \sum_{q} e_{q}^{2} x_{H} q_{q}^{p}\left(x_{H}, Q^{2}\right) D_{q}^{H^{\prime}}\left(z_{H}, Q^{2}\right)$
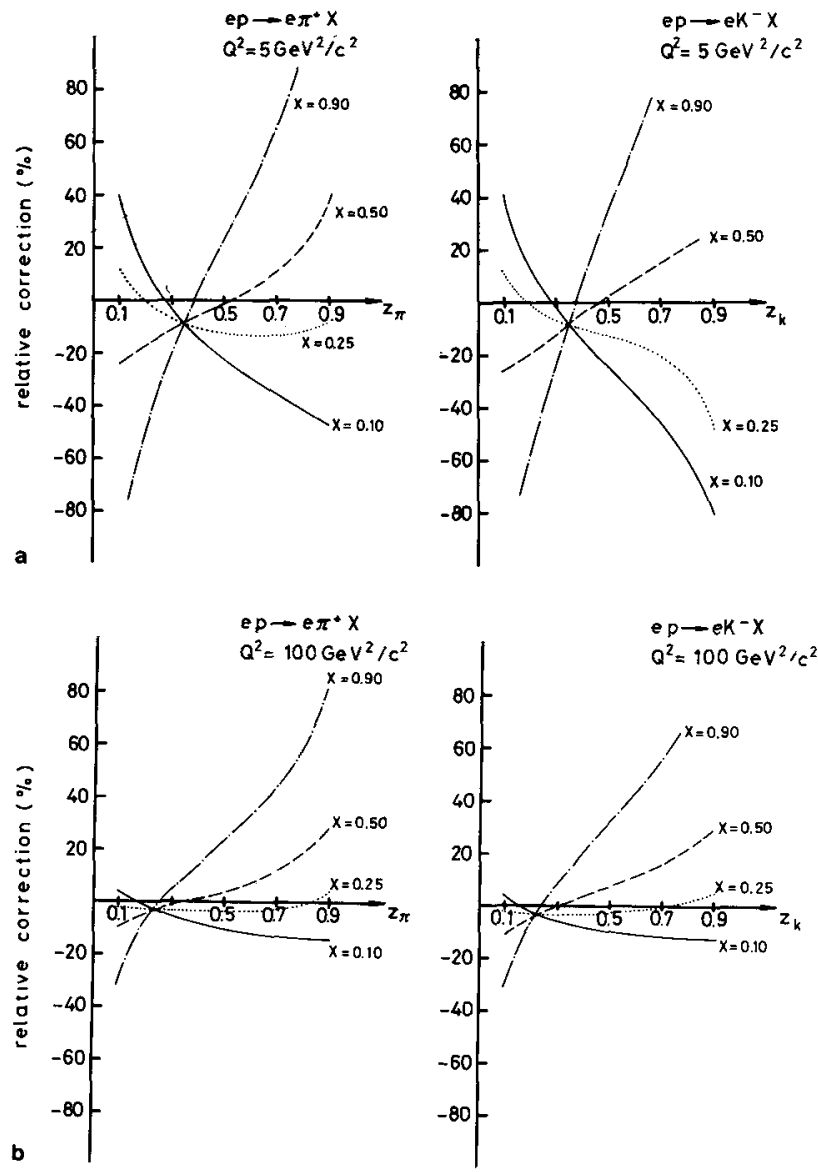

Fig. $5 a$ and b. Relative correction (4.1) to the structure function $F_{2}^{p, H^{\prime}}$ of order $\alpha_{s}\left(Q^{2}\right)$ for the two reactions $e p \rightarrow e \pi^{+}+X$ and $e p \rightarrow e K^{-} X$ as a function of $z_{H}$ for different values of the Bjorken scaling variable $x$ at a $Q^{2}=5 \mathrm{GeV}^{2}$ and $\mathbf{b} Q^{2}=100 \mathrm{GeV}^{2}$ 
for the two reactions $e p \rightarrow e \pi^{+} X$ and $e p \rightarrow e K^{-} X$. The values for $Q^{2}$ chosen are $Q^{2}=5$ and $100 \mathrm{GeV}^{2}$. For the running coupling constant [2] we take $\alpha_{s}\left(Q^{2}\right)=\frac{12 \pi}{25 \ln Q^{2} / \Lambda^{2}}$ and fix the scale parameter by $\Lambda=0.5 \mathrm{GeV}$. The plots are shown as functions of $z_{H}$ for different values of the Bjorken scaling variable $x_{H}\left(x_{H}=0.10,0.25,0.50\right.$ and 0.90$)$. The relative correction for the different secondaries $\pi^{+}$and $K^{-}$is quite similar in shape and magnitude, although the $K^{-}$ has no common valence quarks with the proton. In both cases there is the expected decrease with increasing $Q^{2}$, but for the large $x_{H}$ region, $x_{H} \gtrsim 0.5$, the decrease is slow. At low $Q^{2}\left(Q^{2}=5 \mathrm{GeV}^{2}\right)$ Fig. 5a indicates non-negligible corrections of the order of $20-60 \%$ for $x_{H} \simeq 0.10-0.25$ and $z_{H}>0.5$, which may be measured in high statistics electroproduction experiments. For fixed $x_{H}$ the change in sign of the correction term when $z_{H}$ is varied is related to the constraint due to the energy sum rule (3.27). The contributions from the terms in (3.24), which are proportional to the gluon structure function are only significant for $x_{H}<0.2$; the main contribution due to the gluon fragmentation is found in the region $z_{H} \lesssim 0.3$.

In order to estimate the violation of factorization of the semi-inclusive cross-section with respect to its $x_{H^{-}}$ and $z_{H}$-dependence due to the $\alpha_{s}$-corrections (3.24), (3.26) we consider the process $e p \rightarrow e \pi^{ \pm} X$ and compare our estimates with the data of [15]. This experiment covers the kinematic range of $2<Q^{2}<6 \mathrm{GeV}^{2}$ $\left(\left\langle Q^{2}\right\rangle=2.8 \mathrm{GeV}^{2}\right)$ and $0.1<x_{H}<0.45$. In the quarkparton model the normalized cross-section for charged pion production becomes independent of $x_{H}$, when the region $x_{H}>0.1$ is considered, where valence quarks dominate,

$\frac{1}{d \sigma / d x_{H}}\left[\frac{d \sigma^{\pi^{+}}}{d x_{H} d z_{H}}+\frac{d \sigma^{\pi^{-}}}{d x_{H} d z_{H}}\right] \simeq D_{u}^{\pi^{+}}\left(z_{H}\right)+D_{u}^{\pi^{-}}\left(z_{H}\right)$,

where $u$ denotes the up quark in the proton.

In [15] this prediction of the quark-parton model is tested by plotting the data for the left hand side of (4.2) for three different regions of $x_{H}$, divided by the average over the full $x_{H}$ range at each fixed $z_{H}$ value. This plot is shown in Fig. 6. It is pointed out in [15] that this test is limited by the systematic error associated with the kaon and proton subtractions made in the analysis of this experiment. The curves shown in Fig. 6 represent the violation of the factorization property (4.2) when the corrections of order $\alpha_{s}$ are taken into account. Integrating the cross-section $d \sigma / d x_{H} d z_{H} d y$ with respect to $y$ (3.2) we obtain instead of (4.2),

$\frac{1}{d \sigma / d x_{H}}\left[\frac{d \sigma^{\pi^{+}}}{d x_{H} d z_{H}}+\frac{d \sigma^{\pi^{-}}}{d x_{H} d z_{H}}\right] \simeq D_{u}^{\pi^{+}}\left(z_{H}, Q^{2}\right)$

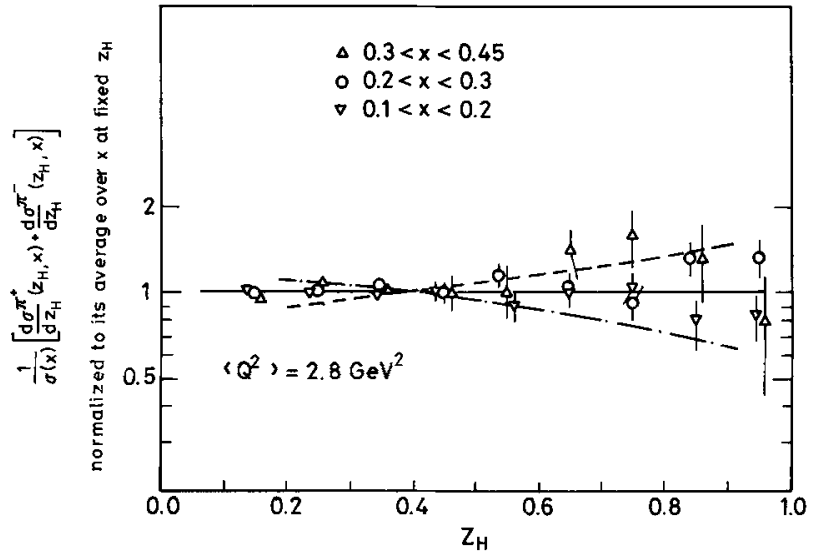

Fig. 6. The cross-section ratio $\frac{1}{d \sigma / d x} \cdot\left[\frac{d \sigma^{\pi^{+}}}{d x d z_{H}}+\frac{d \sigma^{\pi^{-}}}{d x d z_{H}}\right]$ for electroproduction normalized to its average over $x$ at fixed $z_{H}$. The data are from [15]. The curves illustrate the violation of factorization as calculated from (4.3) at $Q^{2}=2.8 \mathrm{GeV}^{2}$ for $x=0.15$ (dasheddotted), $x=0.25$ (solid) and $x=0.37$ (dashed curve)

$$
\begin{aligned}
& +D_{u}^{\pi^{-}}\left(z_{H}, Q^{2}\right)+\left\{\frac { 1 } { F _ { 2 } ^ { p } ( x _ { H } , Q ^ { 2 } ) } \left[F_{\text {corr. }}^{p, \pi^{+}+\pi^{-}}\left(x_{H}, z_{H}, Q^{2}\right)\right.\right. \\
& \left.\left.-\frac{1}{4} x_{H}\left(F_{L}^{p, \pi^{+}+\pi^{-}}\left(x_{H}, z_{H}, Q^{2}\right)-F_{L}^{p}\left(x_{H}, Q^{2}\right)\right)\right]\right\},
\end{aligned}
$$

where the terms in the curly brackets are of order $\alpha_{s}\left(Q^{2}\right)$, and they are evaluated from (3.24) and (3.26). The results are presented in Fig. 6 for $x_{H}=0.15$ (dashed-dotted curve), $x_{H}=0.25$ (solid curve) and $x_{H}=0.37$ (dashed curve) taken at $Q^{2}=\left\langle Q^{2}\right\rangle=$ $2.8 \mathrm{GeV}^{2}$. The contributions from the longitudinal terms in (4.3) are negligible. The deviations from factorization become significant at large values of $z_{H}$, and at $z_{H} \simeq 0.8$ they are predicted to be $20 \%$.

In Fig. 7 the charged pion data from the processes $e p \rightarrow e \pi^{ \pm} X\left(\right.$ at $\left.\left\langle Q^{2}\right\rangle=2.8 \mathrm{GeV}^{2}\right)[15]$ and $e^{+} e^{-} \rightarrow$ $\pi^{ \pm} X[16]$ (multiplied by 0.5 ) at $Q^{2}=13 \mathrm{GeV}^{2}$ are shown as functions of $z_{H}$ for $z_{H} \gtrsim 0.5$. According to the quark-parton model these two distributions are expected to be equal, when the contributions to $e^{+} e^{-}$ from strange quarks are neglected. However, in Fig. 7 one observes in this range of $z_{H}$ that the $\pi^{ \pm}$yield from $e^{+} e^{-}$annihilation is smaller than the one from $e p$ scattering. In the framework of QCD this decrease of the spectra for $z_{H} \gtrsim 0.5$ with increasing $Q^{2}$ is described by the $Q^{2}$-evolution of the fragmentation functions $D_{q}^{\pi \pm}\left(z_{H}, Q^{2}\right)$. This is shown in Fig. 7: the solid curve is the result from (4.3) for the $e p$ reaction evaluated at $x_{H}=0.25$ and $Q^{2}=2.8$ (the dasheddotted curve is calculated without the $\alpha_{s}$-corrections) and the dashed curve is the prediction for $e^{+} e^{-}$ annihilation at $Q^{2}=13 \mathrm{GeV}^{2}(2.14)$. For the case of neutrino scattering we estimate the finite corrections of order $\alpha_{s}$ for the cross-section of the process $v p \rightarrow \mu^{-} \pi^{ \pm} X$. For the sake of simplicity we neglect contributions due to the sea- and charmed quarks. 


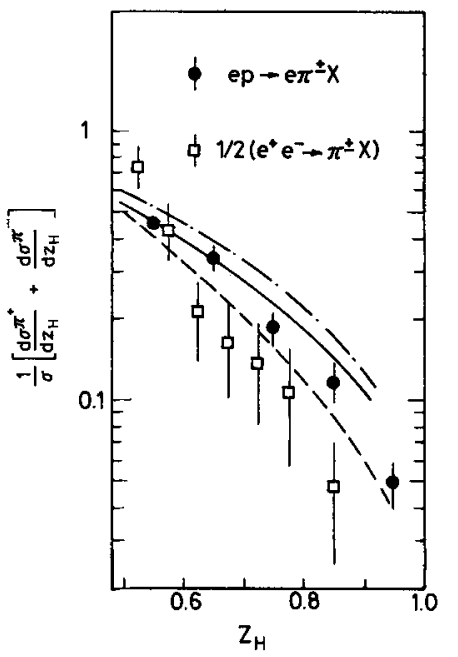

Fig. 7. Distributions for charged pion-production from electroproduction and electron-positron annihilation. The $e-p$ data are from [15] for $2<Q^{2}<6 \mathrm{GeV}^{2},\left\langle Q^{2}\right\rangle=2.8 \mathrm{GeV}^{2}$, the $e^{+} e^{-}$data are from [16] for $Q^{2}=13 \mathrm{GeV}^{2}$. The solid curve is the prediction for $e p \rightarrow e^{\pi \pm} X$ (The dashed-dotted curve is calculated without the $\alpha_{s}$-terms in (4.3)) and the dashed one for $e^{+} e^{-} \rightarrow \pi^{ \pm} X$

In the parton model [1] the normalized cross-section is simply given by

$\frac{1}{d \sigma / d x_{H}} \frac{d \sigma^{\pi}}{d x_{H} d z_{H}}=\frac{F_{2}^{p, \pi^{ \pm}}\left(x_{H}, z_{H}\right)}{F_{2}^{p}\left(x_{H}\right)}=D_{u}^{\pi^{ \pm}}\left(z_{H}\right)$,

which is independent of $x_{H}$. The deviations from this factorization property due to the finite terms of order $\alpha_{s}$ can be expressed by taking the ratio of moments [17]

$$
=\frac{\frac{F_{2, n m}^{\pi^{+}-\pi^{-}}\left(Q^{2}\right)}{F_{2, n}\left(Q^{2}\right)}}{\int_{0}^{1} d x_{H} x_{H}^{n-2} \int_{0}^{1} d z_{H} z_{H}^{m-1} F_{2}^{p, \pi^{+}-\pi^{-}}\left(x_{H}, z_{H}, Q^{2}\right)},
$$

where we consider the difference of $\pi^{+}$and $\pi^{-}$ production. From (3.24) modified for the case of neutrino scattering we obtain

$$
\begin{aligned}
& \frac{F_{2, n m}^{\pi^{+}-\pi^{-}}\left(Q^{2}\right)}{F_{2, n}\left(Q^{2}\right)}=\left(D_{m}^{\pi^{+}}\left(Q^{2}\right)-D_{m}^{\pi^{-}}\left(Q^{2}\right)\right) \\
& \cdot\left[1+\frac{\alpha_{s}\left(Q^{2}\right)}{2 \pi} \frac{4}{3}\left(c_{1}(n, m)+2 \frac{g_{n}\left(Q^{2}\right)}{d_{n}\left(Q^{2}\right)} c_{2}(n, m)\right)\right],
\end{aligned}
$$

where $\quad D_{m}^{\pi^{+}}\left(Q^{2}\right)-D_{m}^{\pi^{-}}\left(Q^{2}\right)=\int_{0}^{1} d z z^{m-1}\left(D_{u}^{n^{+}}\left(z, Q^{2}\right)-\right.$ $\left.D_{u}^{\pi^{-}}\left(z, Q^{2}\right)\right)$ and $g_{n}\left(Q^{2}\right)$ and $d_{n}\left(Q^{2}\right)$ are the moments of the structure functions for the gluon and the $d$-quark in the proton,

$$
g_{n}\left(Q^{2}\right)=\int_{0}^{1} d x x^{n-1} g\left(x, Q^{2}\right), d_{n}\left(Q^{2}\right)=\int_{0}^{1} d x x^{n-1} d\left(x, Q^{2}\right)
$$

The coefficients $c_{1,2}(n, m)$ are

$$
\begin{aligned}
& c_{1}(n, m)=\frac{5}{2}-\sum_{k=1}^{m-1} \frac{1}{k^{2}}-\sum_{k=1}^{m+1} \frac{1}{k^{2}}-\frac{3}{2} \sum_{k=1}^{m} \frac{1}{k}+\frac{1}{2} \frac{1}{m+1} \\
& -\frac{3}{2} \sum_{k=1}^{n-1} \frac{1}{k}-\frac{2}{n}-\frac{3}{n+1}+\frac{1}{m n}+\frac{1}{n} \sum_{k=1}^{m} \frac{1}{k} \\
& +\frac{1}{n+1} \sum_{k=1}^{m-1} \frac{1}{k}+\frac{6}{(n+1)(m+1)} \\
& +\sum_{l=1}^{n-1} \frac{1}{l}\left(\sum_{k=1}^{m-1} \frac{1}{k}+\sum_{k=1}^{m+1} \frac{1}{k}\right), \\
& c_{2}(n, m)=\frac{3}{4}\left[\left(\frac{6}{n+2}-\frac{6}{n+1}+\frac{1}{n}\right)\left(1-\frac{2}{m}\right)\right. \\
& \left.-\left(\frac{2}{n+2}-\frac{2}{n+1}+\frac{1}{n}\right) \sum_{k=1}^{m-2} \frac{1}{k}\right] .
\end{aligned}
$$

Because of the difference $\pi^{+}-\pi^{-}$the terms proportional to the gluon fragmentation function $D_{g}^{\pi \pm}$ drop out. In contrast to the parton model result given above, $F_{2, n m}^{\pi^{+}-\pi^{-}}$does no longer factorize in $x_{H}$ and $z_{H}$ moments separately. The ratio (4.5) remains $n$-dependent, and at fixed $m$ and $Q^{2}$ the $n$-dependence becomes stronger when $n$ is increased. The numerical evaluation of (4.6) predicts that at $Q^{2}=10 \mathrm{GeV}^{2}$ the breaking of the factorization is less than $20 \%$ when the moments with $n \leqq 5$ and $m \leqq 5$ are considered.

The above estimates show that the finite terms of order $\alpha_{s}$ can indeed be treated as corrections to the leading logarithm approximation and a detailed test (e.g. via factorization) needs very precise experiments.

\section{References}

1. Feynman, R.P. : Photon-Hadron Interactions Benjamin, 1972

2. Politzer, H.D. : Phys. Rep. 14C, 129 (1974); Marciano, W., Pagels, H. : Phys. Rep. 36C, 137 (1978); Politzer, H.D.: Talk presented at the XIX International Conference on High Energy Physics, Tokyo, 1978

3. Ellis, R.K., Georgi, H., Machacek, M., Politzer, H.D., Ross, G.G. : Phys. Lett. 78B, 281 (1978) and preprint CALT 68-684 (1978). These papers contain references to related literature

4. Altarelli, G., Parisi, G. : Nucl. Phys. B126, 298 (1977)

5. Georgi, H., Politzer, H.D. : Nucl. Phys. B136, 445 (1978)

6. Owens, J.F. : Phys. Lett. 76B, 85 (1978);

Uematsu, T. : Phys. Lett. 79B, 97 (1978)

7. Altarelli, G., Ellis, R.K., Martinelli, G., Nucl. Phys. B143, $521(1978)$

8. Kubar-André, J., Paige, F.E. : Phys. Rev. D8, 221 (1979); Contogouris, A.P., Kripfganz, J. : Montreal MeGill University preprint (1978):

Abad, J., Humpert, B. : Phys. Lett. 78B, 627 (1978);

Harada, K., Kaneko, T., Sakai, N. : preprint CERN TH-2619 (1979)

9. Sakai, N. : preprint CERN TH-2641 (1979)

10. Sterman, G., Weinberg, S. : Phys. Rev. Lett. 39, 1436 (1977); Basham, C.L., Brown, L.S., Ellis, S.D., Love, S.T.: Phys. Rev. D17, 2298 (1978) 
11. Ellis, J., Gaillard, M.K., Ross, G. : Nucl. Phys. B111, 253 (1976);

De Grand, T.A., Ng, Y.J., Tye, S.-H.H.: Phys. Rev. D16, 3251 (1977)

12. Appelquist, T., Georgi, H. : Phys. Rev. D8, 4000 (1973); Zee, A. ; Phys. Rev. D8, 4038 (1973)

13. Méndez, A. : Nucl. Phys. B145, 199 (1978)
14. Baier, R., Engels, J., Petersson, B.: University Bielefeld preprint Bl-TP $79 / 10$

15. Drews, G. et al. : Phys. Rev. Lett. 41, 1433 (1978)

16. Brandelik, R. et al. : Phys. Lett. 67B, 358 (1977)

17. Scott, W.G.: Talk presented at the XIV Rencontre de Moriond, 1979 